\title{
Ternary Copolymerization of Trimethylvinyloxysilane, Styrene, and Acrylic Esters Catalyzed by Organoaluminum Compounds
}

\author{
Tadamichi HIRABAYASHI and Kenji YOKOTA \\ Materials Research Laboratory, Nagoya Institute of Technology, \\ Gokiso-cho, Showa-ku, Nagoya 466, Japan.
}

(Received May 6, 1980)

\begin{abstract}
The ternary copolymerization of trimethylvinyloxysilane, styrene, and methyl or phenyl acrylate was attempted in the presence of organoaluminum compounds at $0^{\circ} \mathrm{C}$. All copolymers had a considerable amount of five-membered lactone structures in their backbone chains following the hydrolysis of the trimethylsiloxy group. These copolymers originally had a $1: 1$ composition between acrylate as an electron acceptor and the other two components as electron donors, and therefore could be recognized as copolymers of two alternating units, trimethylvinyloxysilane-acrylate and styrene-acrylate. The conversion of lactonization was often beyond $86.5 \%$ (Flory's limit) being nearly $100 \%$, especially in copolymers containing the phenyl acrylate component. Additional lactonization of the copolymers did not occur under the same conditions used in the work-up process. Hence, the realized conversion resulted from a statistical intramolecular reaction between trimethylsiloxy and alkoxycarbonyl groups. These terpolymers may thus be regarded as a model of the "specially-designed alternating copolymer" described in the preceding paper.
\end{abstract}

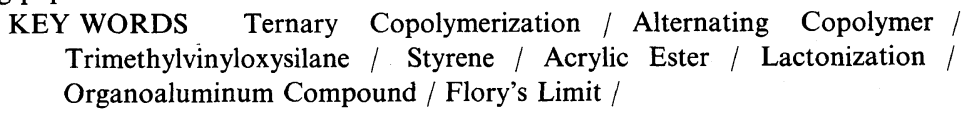

Recently, attention has been directed to the permeation and transport activities of particular ions of membranes of synthetic polymers containing lactone structures. ${ }^{1}$ Such polymers can be synthesized either by polymerizing unsaturated lactone monomers or by reacting two neighboring substituents on a copolymer chain so as to form an ester bond. However, unsaturated lactone monomers often show low polymerizabilities ${ }^{2}$ and the substituents of a simple copolymer are not always arranged in favor of intramolecular lactonization.

Alternating copolymers, whose syntheses have been developed in this decade, ${ }^{3}$ have a favorable arrangement of two substituents for intramolecular reaction. Hirooka et al. ${ }^{4}$ reported the hydrolysis and lactonization of alternating butyl vinyl ether-methyl methacrylate copolymer. Furukawa et al..$^{5}$ reported the lactamization of alternating $N$-vinylsuccinimide-maleic anhydride copolymer with the aim of using the copolymer as a chelating agent.

According to Flory's statistical considerations, ${ }^{6}$ however, even an alternating copolymer can not achieve $100 \%$ conversion. Inevitably, $13.5 \%$ of the substituents remain unreacted, as was experimentally observed in Hirooka's study. ${ }^{4}$

In the preceding paper, ${ }^{7}$ the authors showed theoretically that the substituents of a speciallydesigned alternating copolymer could undergo $100 \%$ intramolecular reaction beyond the so-called Flory's limit. In order to show an experimental example of such a copolymer, we consider trimethylvinyloxysilane to be important. This monomer is quite similar to the polymerization behavior ${ }^{8}$ of the corresponding carbon analogues, vinyl ethers, but is unique because of its lability to $\mathrm{Si}-\mathrm{O}$ bond toward hydrolysis. Thus, in this paper, trimethylvinyloxysilane, styrene, and methyl or phenyl acrylate are ternary copolymerized in the presence of organoaluminum compounds. The hydrolysis of the terpolymer introduces a lactone structure on the derived copolymer. 


\section{EXPERIMENTAL}

\section{Monomers}

Trimethylvinyloxysilane (VOSi) was prepared according to Nesmeyanov's method. ${ }^{9}$ Careless distillation of the crude product resulted in the formation of hexamethyldisiloxane and divinyl ether. The trap-to-trap technique under reduced pressure $(\sim 100 \mathrm{mmHg})$ was preferable as a purification method. bp $74-75^{\circ} \mathrm{C}(760 \mathrm{mmHg})$; NMR $\left(\mathrm{CDCl}_{3}\right) \delta 0.23(\mathrm{~s}, 9), 4.00(\mathrm{~d}, 1, J=13.5 \mathrm{~Hz}$, trans$\left.\mathrm{CH}_{2}=\mathrm{CH}\right), 4.28\left(\mathrm{~d}, 1, J=6.0 \mathrm{~Hz}\right.$, cis $\left.-\mathrm{CH}_{2}=\mathrm{CH}\right)$, and $6.25 \mathrm{ppm}$ (d-d, 1, =CH-); purity, $>\overline{96.5 \%}$.

Phenyl acrylate (PhA) was prepared by the reaction of acrylic chloride and sodium phenoxide in water. A repeated distillation of the crude product gave a pure fraction. bp $57.0-57.5^{\circ} \mathrm{C}(1.5$ $\mathrm{mmHg}) ; d_{4}^{20} 1.055$ [lit. ${ }^{10}$ bp $59-60^{\circ} \mathrm{C}(1.5 \mathrm{mmHg})$, $\left.d^{20} 1.076\right]$.

Styrene (St) and methyl acrylate (MA) were obtained commercially, purified as usual, and distilled under nitrogen.

\section{Other Reagents}

Catalysts, diethylaluminum chloride $\left(\mathrm{Et}_{2} \mathrm{AlCl}\right)$, and ethylaluminum sesquichloride $\left(\mathrm{Et}_{3} \mathrm{Al}_{2} \mathrm{Cl}_{3}\right)$ were available from Ethyl Corp., distilled under nitrogen, and used as $4 \mathrm{moll}^{-1}$ toluene solutions.

Toluene was washed in concd sulfuric acid and water, dried over sodium wire, and distilled.

\section{Copolymerization}

All reagent feeding procedures were performed under an argon atmosphere at $-78^{\circ} \mathrm{C}$. The toluene solution of acrylate monomer was placed in a dry test tube and a calculated volume of catalyst solution was added. After $30 \mathrm{~min}$, St and VOSi were added gently. The test tube was then sealed off and dipped in a thermostated bath at $0^{\circ} \mathrm{C}$.

After a desired period, the content of the tube was poured into cold methanol containing $1 \mathrm{vol} \%$ of concd hydrochloric acid. The precipitated copolymer was collected and reprecipitated twice from a chloroform solution with methanol. Dimethyl sulfoxide (DMSO) was used instead of chloroform for certain samples having poor solubility. When only a trace of copolymer sample was recovered by the above procedure, the methanol was evaporated and the residue was reprecipitated from a DMSO solution with water. All copolymer samples were dried in vacuo at $55^{\circ} \mathrm{C}$.
Analyses

Nuclear magnetic resonance (NMR) spectra on a Hitachi R-20B or R-24A spectrometer and infrared (IR) spectra on a JASCO IR-DG-701G spectrometer were taken. Elemental analysis was carried out by Yanagimoto CHN-MT-1S apparatus. Molecular weight was estimated by gel-permeation chromatography (GPC) in tetrahydrofuran on a Toyo Soda HLC-802UR model equipped with TSK$\mathrm{G} 5000 \mathrm{H}_{8}$ and $-\mathrm{G} 2500 \mathrm{H}_{8}$ columns and detected by ultraviolet at $254 \mathrm{~nm}$ and also by differential refractometry.

\section{RESULTS AND DISCUSSION}

\section{Ternary Copolymerization of VOSi, St, and MA}

Table I summarizes the results of the ternary copolymerization of $\mathrm{VOSi}$, St, and MA in the presence of $\mathrm{Et}_{3} \mathrm{Al}_{2} \mathrm{Cl}_{3}$ at $0^{\circ} \mathrm{C}$. In the absence of the organoaluminum catalyst, the monomer mixture did not undergo any appreciable polymerization.

The IR and NMR spectra of obtained copolymers showed the absence of any trimethylsiloxy groups. The precipitation procedure with acidic methanol apparently effected the complete hydrolysis of this group. The IR spectra did show, however, the presence of hydroxy and fivemembered lactone groups as well as methoxycarbonyl and phenyl groups. Figure 1 shows that, as both absorptions at $3400 \mathrm{~cm}^{-1}\left(v_{\mathrm{OH}}\right)$ and $1775 \mathrm{~cm}^{-1}$ $\left(v_{\mathrm{C}=0}\right.$, five-membered lactone) increased in intensity, the absorption at $1725 \mathrm{~cm}^{-1}\left(v_{\mathrm{C}=0}\right.$, methoxycarbonyl) decreased in intensity.

In the typical NMR spectrum of a copolymer in deuterochloroform at $60^{\circ} \mathrm{C}$, four distinguishable absorptions appeared at $7.6-6.7,5.0-4.1,3.9-$ 3.4 , and above $3.4 \mathrm{ppm}$ from TMS. These should be assigned to $\mathrm{C}_{6} \mathrm{H}_{5^{-}},>\mathrm{CH}-\mathrm{O}-\mathrm{C}(=\mathrm{O})-,-\mathrm{COOCH}_{3}$ $+>\mathrm{CH}-\mathrm{OH}$, and the other backbone $\mathrm{CH}_{2}$ and $\mathrm{CH}$ protons, respectively. The NMR spectra in DMSO $-d_{6}$ and pyridine- $d_{5}$ were virtually the same as that in deuterochloroform.

Thus, the copolymer structure may be represented by the following formula.

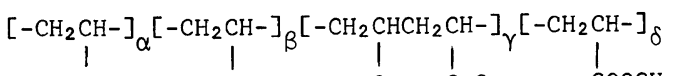

$$
\begin{aligned}
& \mathrm{C}_{6} \mathrm{H}_{5} \quad \mathrm{OH} \quad \mathrm{O}-\mathrm{C}=\mathrm{O} \quad \mathrm{COOCH}_{3}
\end{aligned}
$$

The Greek letters denote the mole fraction of the respective units determined by NMR and elemental analyses and are given in Table I. Evaluations of the 
Ternary Copolymerization of Vinyloxysilane

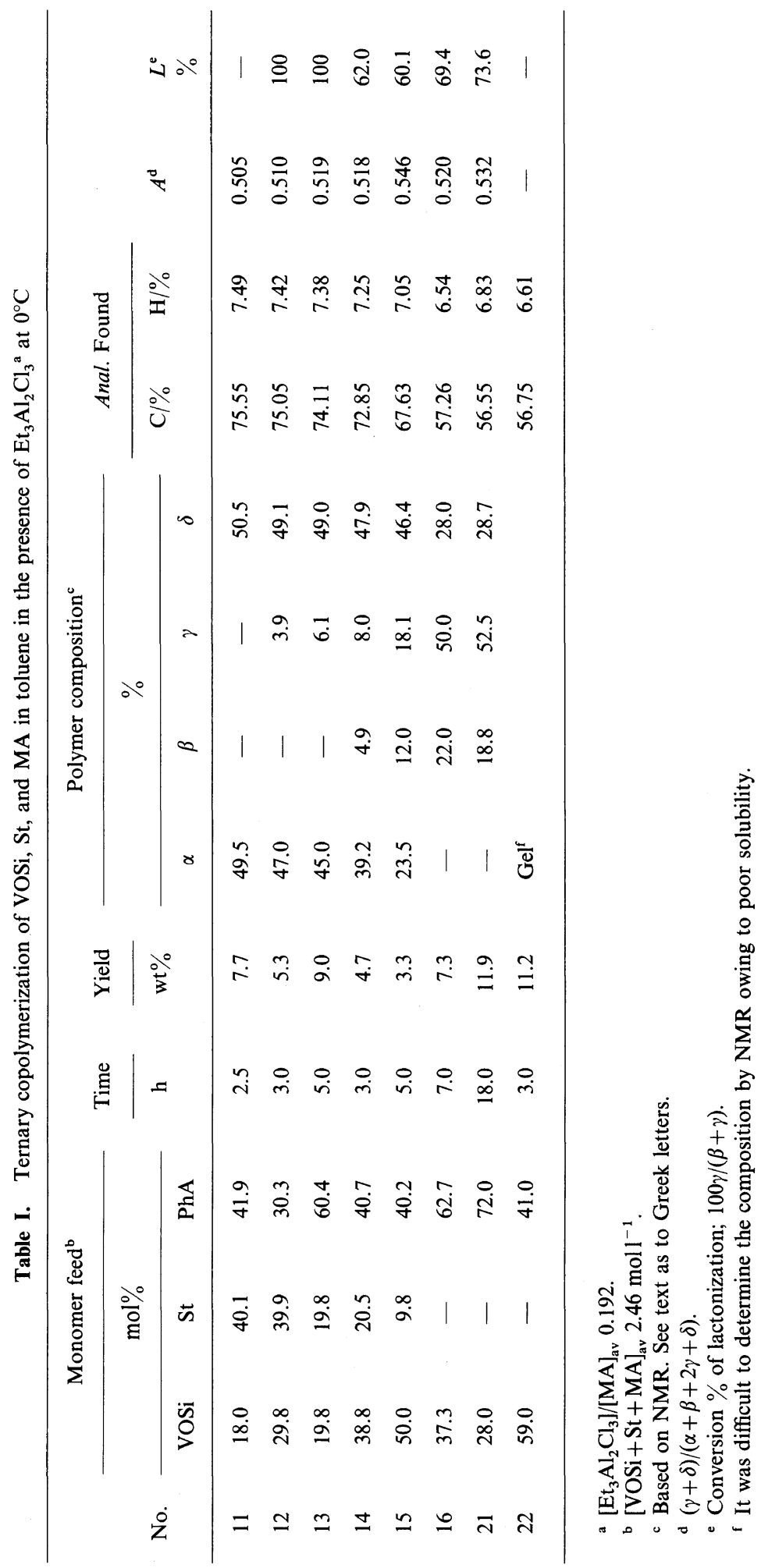




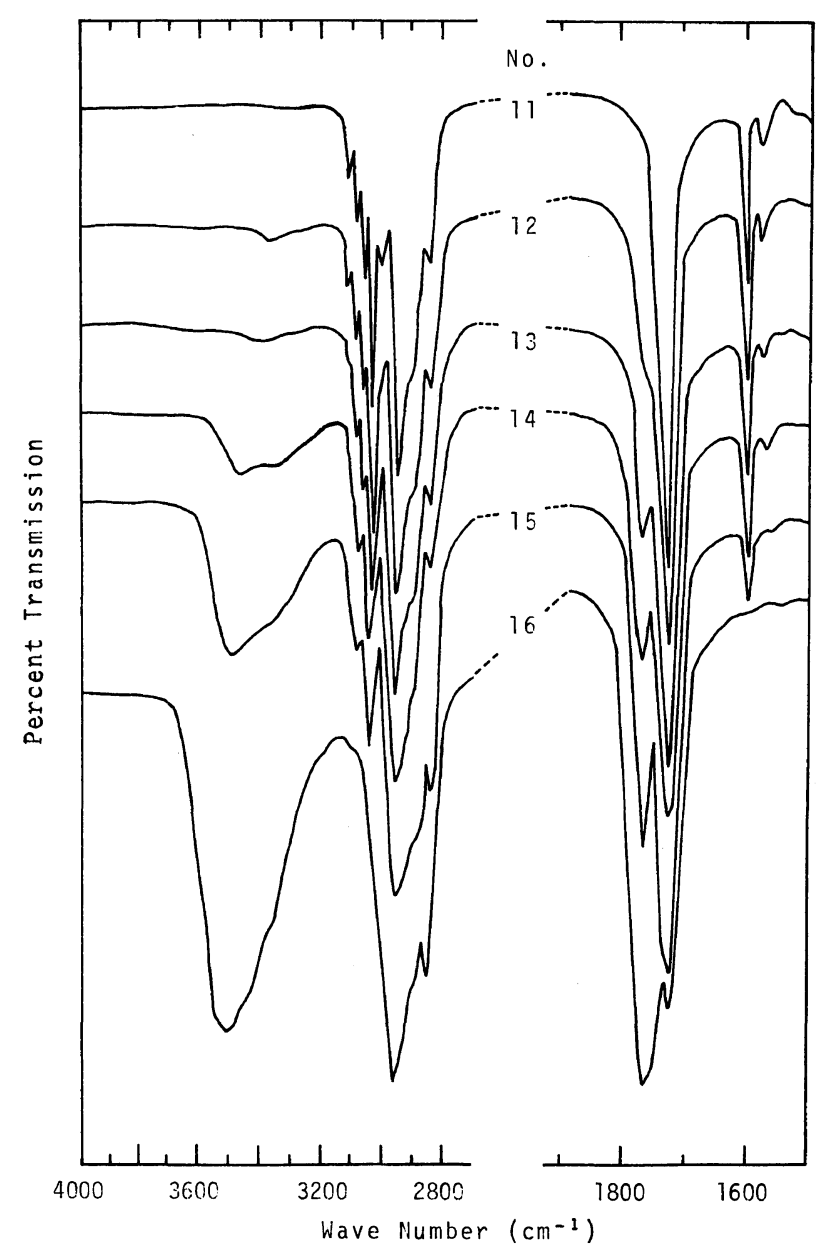

Figure 1. IR spectra of VOSi, St, and MA copolymers.

copolymer composition by NMR and by elemental analysis were found to agree to each other. The former evaluation, however, was sometimes disturbed by small amounts of hydrated water. In fact, the two samples obtained by reprecipitating with water should be considered as hydrated in order to find good agreement between NMR and elemental analyses. Thus, samples No. 16 and 21 in Table I contain 4.17 and $4.09 \mathrm{wt} \%$ of water, respectively.

The mole fraction of MA incorporated originally in the copolymer should be given by the parameter $A=(\gamma+\delta) /(\alpha+\beta+2 \gamma+\delta)$. The parameter $A$ in Table I is essentially close to 0.5 regardless of the various monomer feeds. This fact demonstrates that, in the ternary copolymerization of $\mathrm{VOSi}, \mathrm{St}$, and MA, the former two monomers behave as electron-donating monomers and the MA$\mathrm{Et}_{3} \mathrm{Al}_{2} \mathrm{Cl}_{3}$ complex behaves as an electron-accepting monomer resulting in a copolymer in which the VOSi or St and MA units are alternatingly linked.

It is known that the homopolymerization of VOSi in the presence of cationic catalysts takes place. ${ }^{11}$ In the present case, however, the homopolymer of VOSi must be removed during the work-up process since it can easily be converted into poly(vinyl alcohol) which is soluble in both methanol and water.

The mean molecular weight of the copolymers obtained was roughly estimated by a GPC measurement of their THF solutions. The elution volume giving the maximum amount of copolymers eluted 


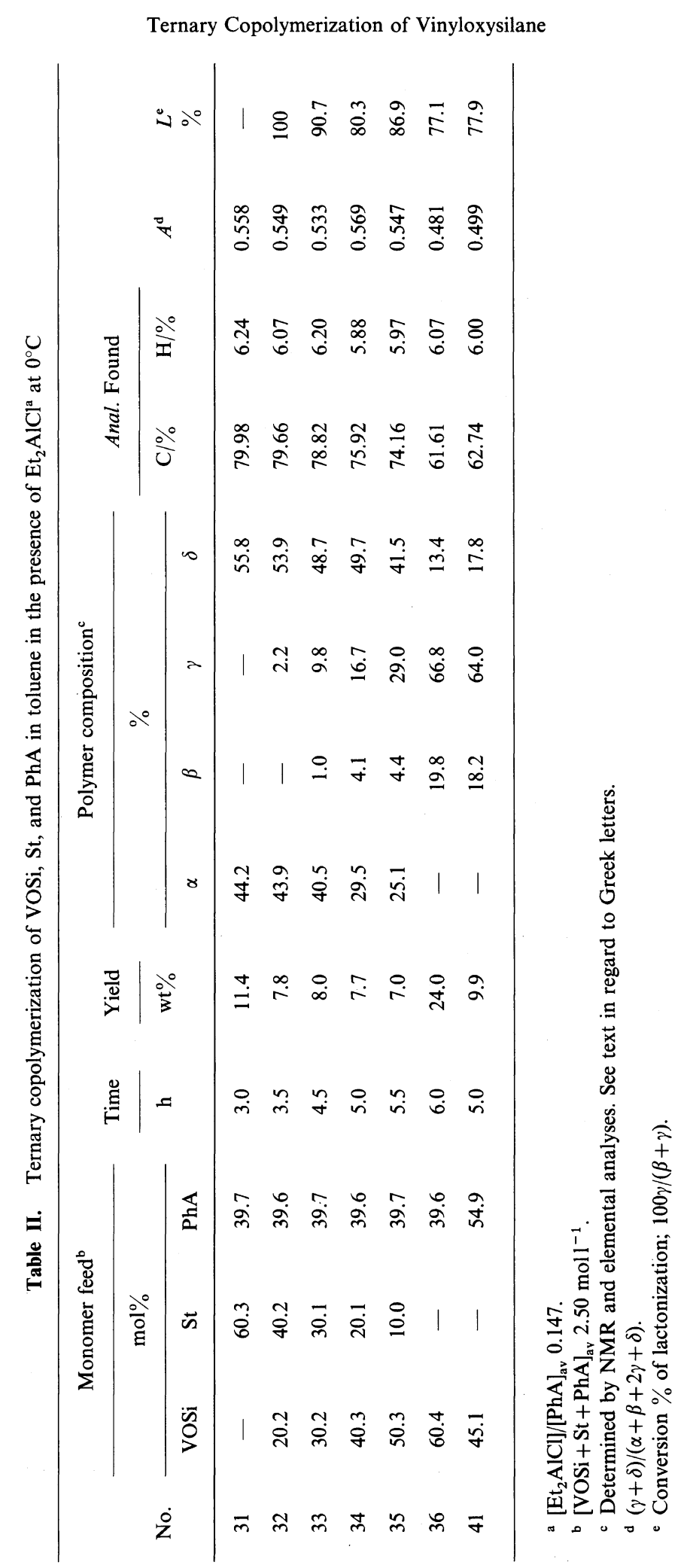

Polymer J., Vol. 13, No. 1, 1981 
corresponded to $(1.5-2.5) \times 10^{5}$ for the weightaveraged molecular weight $\left(M_{w}\right)$ of the poly $(\mathrm{St})$ standard. The data suggest at least that the $M_{w}$ of these copolymers lies in the range of $(0.2-$ $5.5) \times 10^{5}$.

\section{Ternary Copolymerization of VOSi, St, and PhA}

Table II summarizes the ternary copolymerization of VOSi, St, and $\mathrm{PhA}$ catalyzed by $\mathrm{Et}_{2} \mathrm{AlCl}$ at $0^{\circ} \mathrm{C}$.

The IR spectra in Figure 2 show that the copolymer structure can be also given by the following formula.

$\left.\underset{\mathrm{C}_{6} \mathrm{H}_{5}}{\left[-\mathrm{CH}_{2} \mathrm{CH}-\right]_{\alpha}}\right|_{\mathrm{OH}} ^{\left[-\mathrm{CH}_{2} \mathrm{CH}-\right]_{\beta}}{ }_{\beta}^{\left[-\mathrm{CH}_{2} \mathrm{CHCH}_{2} \mathrm{CH}-\right]_{\gamma}}||_{\mathrm{C}=0}^{\left[-\mathrm{CH}_{2} \mathrm{CH}-\right]_{\delta}} \mid \mathrm{COOC}_{6} \mathrm{H}_{5}$

Though the carbonyl stretching vibration seems to be a single absorption for all copolymer samples, the peak-top position shift from $1760 \mathrm{~cm}^{-1}$ (phenoxycarbonyl) to $1772 \mathrm{~cm}^{-1}$ (mostly five-membered lactone) as the copolymer composition changes.

The copolymer composition, determined by NMR and elemental analyses, is listed in Greek letters in Table II. For samples No. 36 and No. 41, a small amount of hydrated water is necessary for obtaining reasonable results. This water can be observed in NMR as sharp peaks at $3.31 \mathrm{ppm}$ in DMSO- $d_{6}$ or $4.61 \mathrm{ppm}$ in pyridine- $d_{5}$.

The mole fraction of $\mathrm{PhA}$ incorporated originally in the copolymer can be presented again in terms of $A=(\gamma+\delta) /(\alpha+\beta+2 \gamma+\delta)$. Though all $A$ parameters are near 0.5 , the $\mathrm{PhA}$ units are approximately $5 \mathrm{~mol} \%$ excess for samples No. $31-35$. Behavior such as this is similar to that found in the St-phenyl methacrylate copolymerization with $\mathrm{Et}_{2} \mathrm{AlCl}^{12}$ $\mathrm{PhA}$ alone polymerizes slowly with $\mathrm{Et}_{2} \mathrm{AlCl}$, as does

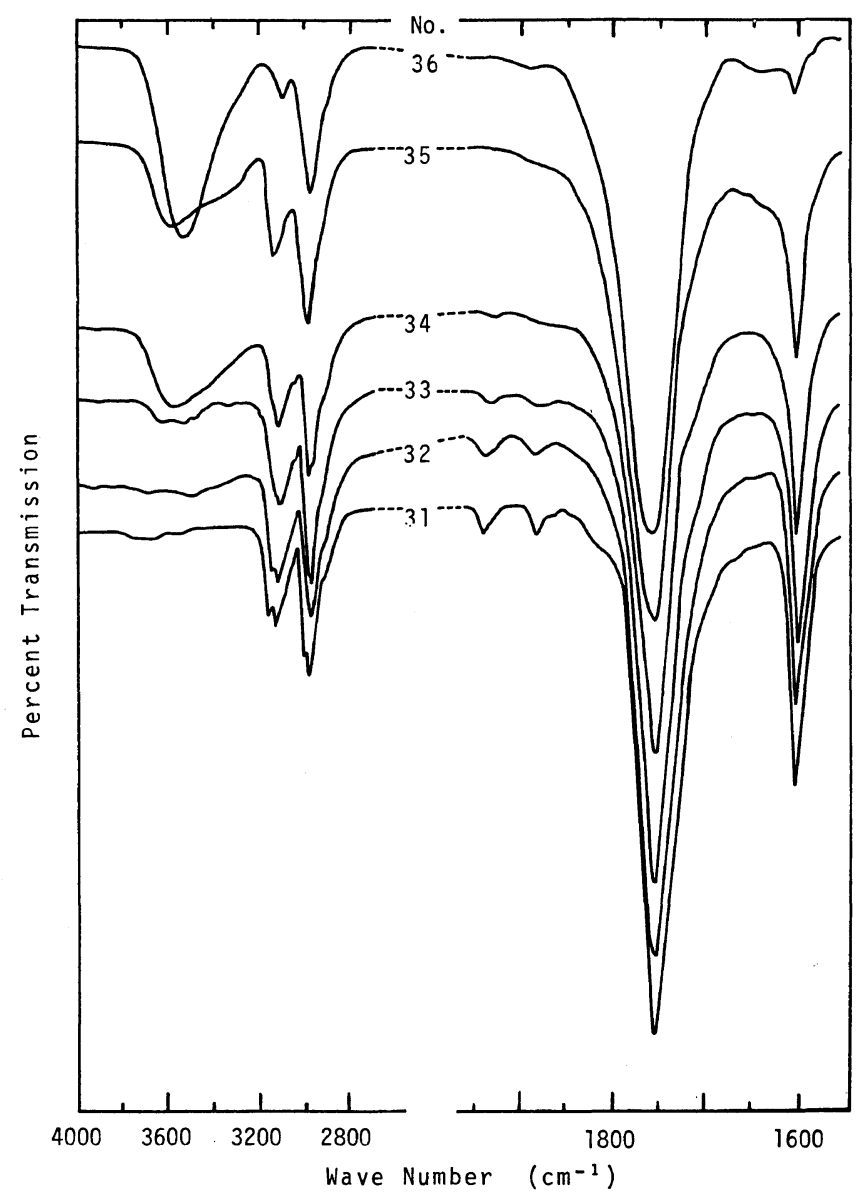

Figure 2. IR Spectra of VOSi, St, and PhA copolymers. 
phenyl methacrylate, but MA does not.

A rough estimation of the mean molecular weight of the VOSi-St-PhA copolymers by GPC data showed $M_{w}$ being in a range of $(1.0-8.5) \times 10^{4}$ for soluble parts in THF.

\section{Lactonization}

The last column of Tables I and II give the lactonization percentage $L$, defined by $L=$ $100 \gamma /(\beta+\gamma)$. The high reactivity of the trimethylsiloxy group toward hydrolysis also brought about lactonization, by a reaction with neighboring alkoxycarbonyl groups on the copolymers. Such lactonization did not occur when simple organic vinyl ethers were used instead of VOSi. The phenoxycarbonyl group showed a lactonization percentage higher than the methoxycarbonyl group, as was expected. ${ }^{13}$ However, it is ambiguous as to whether lactonization occurred during the polymerization or during the work-up operations.

Since the work-up process took place under rather mild conditions, it is unlikely that translactonization occurred during this process. In fact, pulverized samples No. 35 and No. 36 were stirred with methanol containing $1 \mathrm{vol} \%$ concd hydrochloric acid for a week at room temperature, but no additional lactonization occurred, judging from NMR and elemental analyses. Therefore, the present conversions of lactonization should be considered as resulting from statistical intramolecular reactions between trimethylsiloxy and alkoxycarbonyl groups.

As was shown theoretically in the preceding papers, ${ }^{7}$ the intramolecular reactions between $D_{1}$ and $\mathrm{A}$ units of the specially-designed alternating copolymer of $D_{1}, D_{2}$, and $A$ monomers are capable of exceeding the Flory's limit. In this paper, we carried out the ternary copolymerization of VOSi, St, and MA or PhA and the hydrolysis and lactonization of VOSi units of the copolymers. The lactonization could occur beyond the Flory's limit at various mole fractions of VOSi unit. A few VOSiSt-MA samples, No. 14 and No. 15, could not achieved such a satisfactory lactonization and the cause for this may be the low reactivity of methoxycarbonyl groups and uneffective conditions under which even statistically possible pairs of VOSi and MA units could not always react. Another possible explanation for this may be the con-

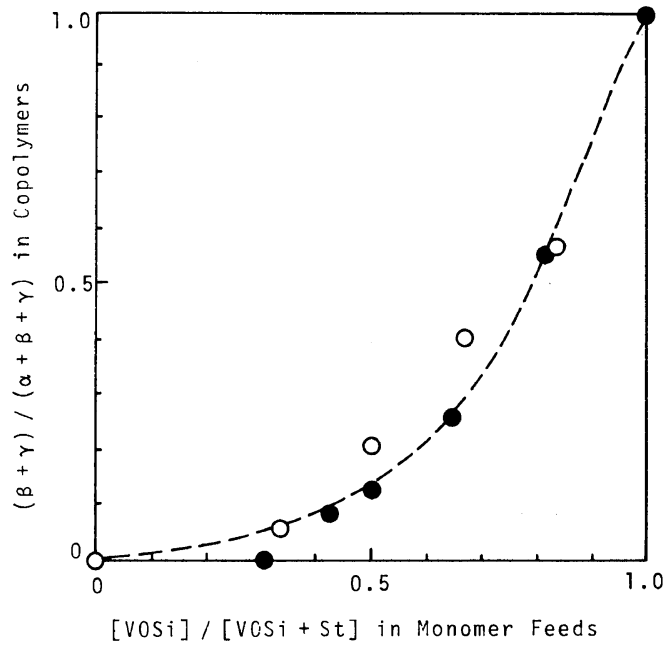

Figure 3. Plots of the parameter $(\beta+\gamma) /(\alpha+\beta+\gamma)$ in copolymers $v s$. [VOSi]/[VOSi $+\mathrm{St}$ ] in monomer feeds: $\bullet$, VOSi-St-MA system; $\mathrm{O}, \mathrm{VOSi}-\mathrm{St}-\mathrm{PhA}$ system. For the dotted line, see text. $\left(r_{\mathrm{VOSi}-\mathrm{MA}}=0.63, r_{\mathrm{St}-\mathrm{MA}}=9.0\right)$.

figuration or conformation of these copolymers. However, there is not helpful data at the present to clarify this point.

Figure 3 shows the dependence of the $(\beta+\gamma) /(\alpha+\beta+\gamma)$ ratio in the copolymer on [VOSi]/[VOSi $+\mathrm{St}]$ in the monomer feed. This is similar to the composition-diagram of copolymer $v s$. monomer feed in a simple copolymerization. Taking into account the existence of the donor-acceptor complexes noticed so well in such an alternating copolymerization, we can formally estimate the "pseudo reactivity ratios" of complexed monomer couples, VOSi-MA or PhA and St-MA or PhA, in the same manner for determining the monomerreactivity ratios, $r_{1}$ and $r_{2}$, and consequently the values $r_{\mathrm{VOSi}-\mathrm{MA}}=0.63$ and $r_{\mathrm{St}-\mathrm{MA}}=9.0$ were obtained from the dotted line in Figure 3. These values suggest that the distribution manner of the two structural units in the VOSi-St-MA terpolymer may be, on the whole, rather block ones but that the VOSi-MA unit tends to make its succesive linkage short enough to cause high conversion of lactonization.

Thus, the terpolymers in this paper can be regarded as models suitable for a "speciallydesigned alternating copolymer" presented theoretically in the preceding paper. 
Acknowledgement. The authors wish to thank Mr. H. Hata of Nagoya Paints Co. Ltd. for making measurements of gel-permeation chromatographs.

\section{REFERENCES}

1. T. Shimizu, M. Yoshikawa, M. Hasegawa, and H. Chiba, Kobunshi Ronbunshu, 34, 753 (1977).

2. Y. Iwakura, T. Tamikado, Y. Fujimoto, S. Ikegami, and M. Maruyama, Kobunshi Kagaku, 15, 469 (1958).

3. H. Hirai, J. Polym. Sci., Macromol. Rev., 11, 47 (1976).

4. M. Hirooka, K. Takebe, Y. Uno, A. Yamano, and K. Maruyama, Ger. Patent 2065345 (1973).

5. J. Furukawa, E. Kobayashi, and T. Doi, J. Polym. Sci., Polym. Chem. Ed., 17, 255 (1979).
6. P. Flory, J. Am. Chem. Soc., 61, 1518 (1939).

7. T. Hirabayashi and K. Yokota, Polym. J., 13, 51 (1981).

8. S. Murahashi, S. Nozakura, M. Sumi, and R. Ohno, J. Polym. Sci., B, 4, 187 (1966).

9. A. N. Nesmeyanov, I. F. Lutsenko, and V. A. Brotsev, Dokl. Akad. Nauk SSSR, 128, 551 (1959); Chem. Abstr., 54, 7537i (1960).

10. P. L. Magagnini and G. Pizzirani, Gazz, Chim. Ital., 96, 1035 (1966); Chem. Abstr., 66, 55154w (1967).

11. S. Nozakura, M. Kitamura, and S. Murahashi, Polym. J., 1, 736 (1970).

12. K. Yokota and T. Hirabayashi, J. Polym. Sci., Polym. Chem. Ed., 16, 2077 (1978).

13. H. Gaeniffey and G. P. Pinazzi, Makromol Chem., 148, 93 (1971). 